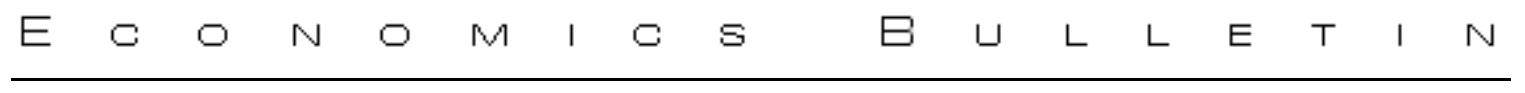

\title{
Strategic Manipulation on Emissions Trading Banking Program with Fixed Horizon
}

\author{
Julien Chevallier \\ EconomiX-CNRS and University Paris 10
}

\begin{abstract}
This paper considers a permit market with both spatial and intertemporal trading. The intertemporal market allows firms to freely borrow or bank permits over a pre-specified period of time. When this period is over, the permit bank has to be balanced, so firms cannot avoid compliance just by borrowing from the future. Market power is introduced by assuming a large dominant agent in a Stackelberg position and a large number of small firms who are nonstrategic but forward looking. The equilibrium is characterized by for the monopoly case and for intermediate cases.
\end{abstract}

\footnotetext{
The author is grateful to Slim Bridji, Denny Ellerman, Pierre-Andre Jouvet and Gilles Rotillon for discussions on this topic, as well as participants to the Emissions Trading Seminar (University of Paris 1, 2006), the IV. Workshop "Permit Trading in Different Applications" (University of Halle Wittenberg, Germany, 2006), the XXIV. Applied Microeconomics Conference (University of Fribourg, Switzerland, 2007), the XV. EAERE Annual Conference (University of Macedonia, Thessaloniki, Greece, 2007) and the LXVI. French Economic Association Meeting (2007) for useful comments and suggestions.

Citation: Chevallier, Julien, (2008) "Strategic Manipulation on Emissions Trading Banking Program with Fixed Horizon."

Economics Bulletin, Vol. 17, No. 14 pp. 1-9

Submitted: May 15, 2008. Accepted: July 28, 2008.

URL: http://economicsbulletin.vanderbilt.edu/2008/volume17/EB-08000014A.pdf
} 


\section{Introduction}

This article builds upon the seminal work by Hahn (1984) which points out the risk of market power and associated economic inefficiencies due to the initial allocation of emission rights (opposite to the standard textbook Coase theorem). It uses an intertemporal framework to focus on the implications of initial allocations allowing for banking and borrowing of permits. As the market imperfection arises during the free permits distribution on the basis of recent emissions ${ }^{1}$, the central part of the paper is an analytical model of a Stackelberg game with a large potentially dominant firm and a competitive fringe. Compared to previous literature, the contribution of this article is threefold. First, the mark-up expression developed throughout the model generalizes in a dynamic context the analysis by Hahn (1984) on the role of initial permits allocation $^{2}$. Second, the results extend Liski and Montero (2005) by explicitly modelling a game structure with hierarchical play and by taking into account the borrowing provision of emission rights. Third, compared to Maeda (2003), the price distortion condition is obtained without loss of generality ${ }^{3}$. The remainder of the article is organized as follows. Section 2 details the model. Section 3 derives a mark-up expression from a benchmark. Section 4 solves the fringe problem taking the action path of the leader as given. Section 5 solves the leader's problem. Section 6 concludes.

\section{Model}

First, I explain the design of the cap-and-trade program. Second, I examine the industry and information structures. Third, I define the intertemporal emissions trading constraint. Fourth, I express the Hotelling conditions. Fifth, I explicit the properties of the abatement cost function.

\subsection{Design of the Cap-and-Trade Program}

The regulator sets a cap $\bar{E}$ on emissions of a given pollutant that corresponds to a specific environmental goal. The fix endowment is exogenous to the model, and may be broken down into individual permits allocation $\bar{e}_{i}$ mandatory for each agent $i$. Agents are further decomposed into two types:

1. agent $\{i=1\}$ is a large polluting agent, who is initially allocated a large number of permits;

2. agents $\{i=2, \ldots N\}$ aggregate many small polluting agent, who are assumed to be comparatively smaller permits-holders, and belong to the competitive fringe.

The competitive market price is determined by fringe agents' abatement costs. The premise of the paper is that the large agent may be able to exert market power.

\subsection{Industry Structure}

This partial equilibrium model features an intra-industry permit market. Market power is defined by Burniaux (1999) as the capacity of an agent to influence the transaction price of traded permits. Thus, I do not

\footnotetext{
${ }^{1}$ See Ellerman and Sue Wing (2003) for a review concerning the use of projections, benchmarking and intensity targets.

${ }^{2}$ Another difference with Hahn's analytical framework lies in the presence of a large number of participants due to the atomicity of fringe agents.

${ }^{3}$ i.e. without specifying a functional form for the abatement cost function. Besides, Maeda neither considers a market power condition within a hierarchical game structure nor examines the effects of introducing banking and borrowing on permits prices.
} 
address exclusionary manipulation strategies ${ }^{4}$ that occur when the dominant agent uses its market power on the permits market to raise entry barriers or exclude agents on the output market.

\subsection{Information Structure}

The large agent who, due to market imperfections induced by initial allocation, receives an amount of permits in excess of his actual emissions needs has a clear incentive to anticipate correctly fringe agents' behaviour. Hence, I investigate the emergence of permits price manipulation strategies through a Stackelberg game with hierarchical play ${ }^{5}$. I model a differential game ${ }^{6}$ played in continuous time where all players have the possibility of influencing the rate of change of the permits bank through the choice of their current actions. It is therefore assumed that they adopt a Markovian strategy where the state variable for the permits bank shows up in the decision rules. The common knowledge includes the fact that all players need to comply to the environmental constraint exogenously set by the regulator.

\subsection{Intertemporal Emissions Trading}

Agents may bank and borrow permits without restrictions. Let $B_{i}(t)$ be the permits bank, with $B_{i}(t)>0$ in case of banking and $B_{i}(t)<0$ in case of borrowing. Any change in the permits bank is equal to the difference between $\bar{e}_{i}(t)$ and $e_{i}(t)$, respectively agent's $i$ permits allocation and his emission level at time $t$. Setting the initial condition $B_{i}(0)=0$, the banking and borrowing constraint may be written as ${ }^{7}$ :

$$
\dot{B}_{i}(t)=\bar{e}_{i}(t)-e_{i}(t)
$$

\subsection{Hotelling Conditions}

Notwithstanding differences between a permit and an exhaustible resource ${ }^{8}$, it is assumed in the literature that the Hotelling conditions for exhaustible resources must apply on a permits market. Consequently, the terminal and exhaustion conditions are introduced.

Terminal Condition Let $[0, T]$ be the continuous time planning horizon ${ }^{9}$. At time $T$, cumulated emissions must be equal to the sum of each agent's depollution objective and therefore to the global cap $\bar{E}$ set by the regulator $^{10}$ :

$$
\int_{0}^{T} \sum_{i=1}^{N} e_{i}(t) \mathrm{dt}=\sum_{i=1}^{N} \bar{e}_{i}=\bar{E}
$$

\footnotetext{
${ }^{4}$ See Misiolek and Elder (1989).

${ }^{5}$ If a pure monopoly emerges as a consequence of the distortions induced by initial allocation, a single seller may price its ouput at a higher level than its marginal cost of production which in turns threatens the efficiency of the permits market.

${ }^{6}$ See Dockner et al. (2000) for an overview of differential games.

${ }^{7}$ See Rubin (1996).

${ }^{8}$ See Liski and Montero (2006) for a discussion of the differences between a tradable permits and a non-renewable resource.

${ }^{9}$ See Schennach (2000) for an alternative time setting including distinct phases of the US Acid Rain Program.

${ }^{10}$ See also Leiby and Rubin (2001).
} 
Exhaustion Condition At time $T$, there is no more permit in the bank (either stocked or borrowed):

$$
\sum_{i=1}^{N} B_{i}(T)=0
$$

Those conditions ensure that agents gradually meet their depollution objective so that the marginal cost of depollution is equalized in present value over the time period, and the permits bank clears in the end. Note there is typically a truncation problem at the end of the period:

- if $B_{i}(T)>0$, surplus allowances are worthless and agents are wasting permits;

- if $B_{i}(T)<0$, agents need to pay a penalty.

\subsection{Abatement Cost Function}

Let $C_{i}\left(e_{i}(t)\right)$ be the abatement cost function ${ }^{11}$ incurred by agent $i$ in order to comply with his permits allocation $\bar{e}_{i}$. $C_{i}\left(e_{i}(t)\right)$ is defined on $\mathbb{R} \rightarrow \mathbb{R}$ and is of class $C^{2}[0 ; T] . C_{i}\left(e_{i}(t)\right)$ is decreasing and convex in $e_{i}(t)$ with $C_{i}^{\prime}\left(e_{i}(t)\right)<0, C_{i}^{\prime \prime}\left(e_{i}(t)\right)>0$ and $C_{i}\left(e_{i}(0)\right)=0$.

Agent's $i$ marginal abatement costs (MAC) are associated with a one-unit reduction from his emission level $e_{i}$ at time $t$ and are noted $-C_{i}^{\prime}\left(e_{i}(t)\right)>0$. At the equilibrium of a permits market in a static framework ${ }^{12}$, price-taking agents adjust emissions until the aggregated MAC is equal to the price $P$ at time $t$ :

$$
P_{t}=-C_{i}^{\prime}\left(e_{i}(t)\right)
$$

\section{Benchmark}

To evaluate the pure effects of over-allocating permits to the large agent with intertemporal flexibility, the dominant agent receives by assumption all the stock of permits. He sets the price at the level which corresponds to the maximization of the difference between revenues from permits sales and its abatement costs. Fringe agents behave as price takers. The mark-up expression may be derived straightforward when the large agent directly integrates the competitive permits price into his maximization program.

\subsection{Optimization program}

The large agent minimizes its abatement costs with respect to total emissions and behaves as follows:

$$
\left\{\begin{array}{l}
\min _{\left\{e_{i}\right\}_{i=1}^{N}} \int_{0}^{T} e^{-r t}\left\{C_{1}\left(e_{1}(t)\right)+P_{t}\left(e_{1}(t)-\bar{e}_{1}(t)\right)\right\} \mathrm{dt} \\
\int_{0}^{T} e_{1}(t) \mathrm{dt}=\bar{E}-\int_{0}^{T} \sum_{i=2}^{N} e_{i}(t) \mathrm{dt} \\
P_{t}=-C_{i}^{\prime}\left(e_{i}(t)\right) \forall i=2, \ldots, N \\
\left(e_{1}(t)-\bar{e}_{1}(t)\right)=\sum_{i=2}^{N} e_{i}(t)
\end{array}\right.
$$

\footnotetext{
${ }^{11}$ Compared to a situation where profits are unconstrained, abatement costs appear in order to meet the emission cap $\bar{e}_{i}$. See Leiby and Rubin (2001).

${ }^{12}$ See Hahn (1984).
} 
where the expression $\left(e_{1}(t)-\bar{e}_{1}(t)\right)$ represents the number of permits bought $(>0)$ or sold $(<0)$. Both types of agent need to comply to the environmental constraint by adjusting their emissions level and trading permits. In this setting, fringe agents' emissions come from trading with the large agent. I replace the value of $P_{t}$ and $\left(e_{1}(t)-\bar{e}_{1}(t)\right)$ in the objective function and form the Lagrangean with $e_{1}(t)$ and $e_{i}(t)$ as control variables, and $\lambda(t)$ as a multiplier:

$$
L=\int_{0}^{T} e^{-r t}\left\{C_{1}\left(e_{1}(t)\right)-C_{i}^{\prime}\left(e_{i}(t)\right) \sum_{i=2}^{N} e_{i}(t)\right\} \mathrm{dt}+\lambda(t)\left(\bar{E}-\int_{0}^{T} e_{1}(t) \mathrm{dt}-\int_{0}^{T} \sum_{i=2}^{N} e_{i}(t) \mathrm{dt}\right)
$$

The first-order conditions are:

$$
\begin{aligned}
\frac{\partial L}{\partial e_{1}(t)}=0 \quad \Leftrightarrow \quad C_{1}^{\prime}\left(e_{1}(t)\right)-\lambda(t)=0 \\
\frac{\partial L}{\partial e_{i}(t)}=0 \quad \Leftrightarrow \quad-C_{i}^{\prime \prime}\left(e_{i}(t)\right) \sum_{i=2}^{N} e_{i}(t)-C_{i}^{\prime}\left(e_{i}(t)\right)-\lambda(t)=0 \quad \forall i=2, \ldots, N
\end{aligned}
$$

Replacing $\lambda(t)=C_{1}^{\prime}\left(e_{1}(t)\right)$ in (7) and rearranging terms, I get:

$$
-C_{1}^{\prime}\left(e_{1}(t)\right)=P(t)\left(1+\varepsilon_{i} \sum_{i=2}^{N} e_{i}(t)\right)
$$

The large agent's MAC is equal to the competitive permits price plus an element of price distortion $\varepsilon_{i}$ defined as fringe agents' elasticity:

$$
\varepsilon_{i}=\frac{C_{i}^{\prime \prime}\left(e_{i}\right)}{C_{i}^{\prime}\left(e_{i}\right)}=\frac{\frac{\mathrm{d} C_{i}^{\prime}}{\mathrm{d} e_{i}}}{\frac{\mathrm{d} C_{i}}{\mathrm{~d} e_{i}}}=\frac{\mathrm{d} C_{i}^{\prime}}{\mathrm{d} e_{i}} \frac{\mathrm{d} e_{i}}{\mathrm{~d} C_{i}}=\frac{\mathrm{d} C_{i}^{\prime}}{\mathrm{d} C_{i}} \quad \forall i=2, \ldots, N
$$

Thus, permits price manipulation results in higher total abatement costs than under perfect competition. Next, I detail this market power condition.

\subsection{Mark-Up Expression}

In this setting, market power is function of fringe agents' elasticity and of the large agent's number of permits:

$$
\varepsilon_{i} \sum_{i=2}^{N} e_{i}(t)=\varepsilon_{i}\left(e_{1}(t)-\bar{e}_{1}(t)\right)
$$

Due to the convexity assumption and $C_{i}^{\prime}\left(e_{i}(t)\right)<0$, fringe agents' elasticity is negative, and reveals the possibility for the leader to affect negatively fringe agents' behaviour. The large agent's MAC is lower than under perfect competition. Since he enjoys a dominant position and has the ability to influence the permits price, the large agent may be characterized overall as a net gainer and fringe agents as net losers.

\section{Fringe Agents' Reaction Function}

First, I solve the fringe problem taking the action path of the leader as given. Fringe agents choose their optimal emissions level according to the possibility to bank and borrow permits in (1). 
Agent's $i(\forall i=2, \ldots, N)$ cost minimization program may be written as follows:

$$
\left\{\begin{array}{l}
\min _{e_{i}} \int_{0}^{T} e^{-r t}\left\{C_{i}\left(e_{i}(t)\right)+P(t)\left(e_{i}(t)-\bar{e}_{i}(t)\right)\right\} \mathrm{dt} \\
\dot{B}_{i}(t)=\bar{e}_{i}(t)-e_{i}(t) \\
B_{i}(0)=0 \\
C_{i}\left(e_{i}(0)\right)=0 \\
\forall i=2, \ldots, N
\end{array}\right.
$$

I write the corresponding current-value Hamiltonian and first-order optimality conditions:

$$
\begin{gathered}
H\left(B_{i}(t), e_{i}(t), \lambda(t), t\right)=\left\{C_{i}\left(e_{i}(t)\right)+P(t)\left(e_{i}(t)-\bar{e}_{i}(t)\right)\right\}-\lambda(t)\left(\bar{e}_{i}(t)-e_{i}(t)\right) \\
\frac{\partial H}{\partial e_{i}(t)}=0 \Leftrightarrow P(t)=-C_{i}^{\prime}\left(e_{i}(t)\right)+\lambda(t) \\
\dot{B}_{i}(t)=\frac{\partial H}{\partial \lambda(t)}=0 \Leftrightarrow \dot{B}_{i}(t)=\bar{e}_{i}(t)-e_{i}(t) \\
\dot{\lambda}(t)-r \lambda(t)=-\frac{\partial H}{\partial B_{i}(t)}=0, \lambda(T) B_{i}(T)=0
\end{gathered}
$$

It can be infered from (14) that $\lambda(t)=\lambda(0) e^{r t}$. The transversality condition (14) is required to meet the exhaustion condition (3), i.e. to reflect the idea that the bank has no scrap value at the end of the period. I distinguish several cases:

- if $B_{i}(T)>0$ or $B_{i}(T)<0$, then $\lambda(T)=0, \lambda(t)=0, \forall i=2, \ldots, N$ : when fringe agents have a net banking or borrowing position at the end of the period, the reaction function is equal to the static equilibrium condition (4) where fringe agents equalize their MAC with the permits price. As stated earlier, surplus allowances are worthless and agents need to pay a penalty in case of net borrowing;

- if $\lambda(T)>0$, then $B_{i}(T)=0$ and $\lambda(t)>0, \forall i=2, \ldots, N$ : when the constraint on $\lambda$ is binding, this implies a positive shadow price for units in the bank that is cleared. The reaction function is equal to (12);

- if $\lambda(T)<0$, then $B_{i}(T)=0$ and $\lambda(t)<0, \forall i=2, \ldots, N$ : this would imply a negative shadow price for units in the bank when it is cleared. Since this result is not in accordance with the specific purpose of a cap-and-trade program, I do not further comment this case.

I have therefore highlighted two possible forms of fringe agents' reaction function $P(t)=-C_{i}^{\prime}\left(e_{i}(t)\right)$ and $P(t)=-C_{i}^{\prime}\left(e_{i}(t)\right)+\lambda(t)$ depending on a net banking/borrowing position or to a positive value of the costate variable.

\section{Behaviour of the Dominant Agent}

Second, I use the first-order conditions of the fringe problem as the constraints in the leader's problem. I characterize the time paths for the fixed horizon banking program and the mark-up expressions for the dominant agent. 


\subsection{Optimization program}

The large agent adjusts strategically his optimal emissions level according to its initial allocation $\bar{e}_{1}$ as expressed by (2) and the banking borrowing constraint (1). The cost minimization program for agent $\{i=1\}$ is:

$$
\left\{\begin{array}{l}
\min _{e_{1}} \int_{0}^{T} e^{-r t}\left\{C_{1}\left(e_{1}(t)\right)+P_{t}\left(e_{1}(t)-\bar{e}_{1}(t)\right)\right\} \mathrm{dt} \\
\dot{B}_{1}(t)=\bar{e}_{1}(t)-e_{1}(t) \\
\bar{E}=\int_{0}^{T} e_{1}(t) \mathrm{dt}+\int_{0}^{T} \sum_{i=2}^{N} e_{i}(t) \mathrm{dt} \\
B_{1}(0)=0 \\
C_{1}\left(e_{1}(0)\right)=0
\end{array}\right.
$$

I now turn my attention to the first case of fringe agents' reaction function. Details for the second case are provided in the Appendix.

\subsection{Solution for the Leader's Problem}

Replacing $P_{t}$ by (4), the large agent's optimization program becomes:

$$
\left\{\begin{array}{l}
\min _{e_{1}} \int_{0}^{T} e^{-r t}\left\{C_{1}\left(e_{1}(t)\right)-C_{i}^{\prime}\left(e_{i}(t)\right)\left(e_{1}(t)-\bar{e}_{1}(t)\right)\right\} \mathrm{dt} \\
\dot{B}_{1}(t)=\bar{e}_{1}(t)-e_{1}(t) \\
\bar{E}=\int_{0}^{T} e_{1}(t) \mathrm{dt}+\int_{0}^{T} \sum_{i=2}^{N} e_{i}(t) \mathrm{dt} \\
B_{1}(0)=0 \\
C_{1}\left(e_{1}(0)\right)=0 \\
\forall i=2, \ldots, N
\end{array}\right.
$$

Assuming fringe agents are homogenous ${ }^{13}$, I write $\sum_{i=2}^{N} e_{i}(t)=(N-1) e_{i}(t)$ and replace the emissions constraint (2) into the objective function:

$$
\left\{\begin{array}{l}
\min _{e_{1}} \int_{0}^{T} e^{-r t}\left\{C_{1}\left(e_{1}(t)\right)-C_{i}^{\prime}\left(\frac{\tilde{E}-e_{1}(t)}{N-1}\right)\left(e_{1}(t)-\bar{e}_{1}(t)\right)\right\} \mathrm{dt} \\
\dot{B}_{1}(t)=\bar{e}_{1}(t)-e_{1}(t) \\
B_{1}(0)=0 \\
C_{1}\left(e_{1}(0)\right)=0
\end{array}\right.
$$

with $\tilde{E}=\frac{\bar{E}}{T}$. I form the corresponding current-value Hamiltonian with $e_{1}(t)$ as a control variable, $B_{1}(t)$ as a state variable, and $\mu(t)$ as a co-state variable:

$$
H\left(B_{1}(t), e_{1}(t), \mu(t), t\right)=C_{1}\left(e_{1}(t)\right)-C_{i}^{\prime}\left(\frac{\tilde{E}-e_{1}(t)}{N-1}\right)\left(e_{1}(t)-\bar{e}_{1}(t)\right)+\mu(t)\left(\bar{e}_{1}(t)-e_{1}(t)\right)
$$

Assuming the existence of an interior solution, necessary optimality conditions include:

\footnotetext{
${ }^{13}$ The homogeneity on the fringe agents is a stylized assumption that allows to derive helpful analytical results.
} 


$$
\begin{gathered}
\frac{\partial H}{\partial e_{1}(t)}=0: C_{1}^{\prime}\left(e_{1}(t)\right)+\frac{1}{N-1} C_{i}^{\prime \prime}\left(\frac{\tilde{E}-e_{1}(t)}{N-1}\right)\left(e_{1}(t)-\bar{e}_{1}(t)\right)-C_{i}^{\prime}\left(\frac{\tilde{E}-e_{1}(t)}{N-1}\right)-\mu(t)=0 \\
\dot{B}_{1}(t)=\frac{\partial H}{\partial \mu(t)}=0 \Leftrightarrow \dot{B}_{1}(t)=\bar{e}_{1}(t)-e_{1}(t) \\
\dot{\mu}(t)-r \mu(t)=-\frac{\partial H}{\partial B_{1}(t)}=0, \mu(T) B_{1}(T)=0
\end{gathered}
$$

With reference to the transversality condition (18), I conduct the same analysis as in the previous section:

- if $B_{1}(T)>0$, then $\mu(T)=0, \mu(t)=0$ and $B_{i}(T)<0, \forall i=2, \ldots, N$ : net banking by the large agent at the end of the period is compensated by fringe agents's net borrowing;

- if $B_{1}(T)<0$, then $\mu(T)=0, \mu(t)=0$ and $B_{i}(T)>0, \forall i=2, \ldots, N$ : net borrowing by the large agent in terminal period is compensated by fringe agents' net banking.

Both cases of net banking $B_{1}(T)>0$ or borrowing $B_{1}(T)<0$ by the large agent in terminal period imply $\mu(T)=0$ and $\mu(t)=0$. From eq.(16), it is possible to identify a price distortion condition analogous to the market power condition (10):

$$
-C_{1}^{\prime}\left(e_{1}(t)\right)=P(t)\left(1+\frac{1}{N-1} \varepsilon_{i}\left(e_{1}(t)-\bar{e}_{1}(t)\right)\right)
$$

On the left hand side of the equation, I have the large agent's MAC. On the right hand side, I recognize the price distortion as a function of fringe agents' elasticity and the large agent's permits endowment.

In either case where both agents have a net banking or borrowing position in terminal period, the large agent is able to affect negatively fringe agent's MAC through the number of permits he holds in excess of his emissions. This result is similar to the benchmark case where the large agent owns all the stock of permits. In the Appendix, I show that this solution holds for the second case of fringe agents' reaction function.

\section{Conclusions}

This article contributes to the link between distributional aspects and overall efficiency of tradable permits markets. First, it extends Hahn (1984)'s analysis on the distortions induced by initial allocation and market power in a dynamic framework. Second, it builds upon Liski and Montero (2005) by modelling strategic interactions after a Stackelberg game and providing a full characterization of the effects of unrestricted borrowing. Third, it derives mark-up expressions without requiring a functional form for the abatement cost function as in Maeda (2003). The emissions trading banking program with fixed horizon is introduced as an environmental regulation tool to minimize total cost for pollution abatement (Rubin (1996), Schennach (2000)). Thus, the results shall not be misinterpreted as suggesting that allowing banking and borrowing decrease the effiency of the permits market. The model could be extended by the adoption of an intertemporal trading ratio specific to borrowing as discussed by Kling and Rubin (1997) ${ }^{14}$, which associated to full banking stands out as the best configuration offered by intertemporal emissions trading to smooth emissions overtime. It may also be interesting to look at another source of heterogeneity between agents, for instance based on their emissions reduction function ${ }^{15}$.

\footnotetext{
${ }^{14}$ The adoption of a discount rate penalizing borrowing may remove some of the perverse incentives whereby agents concentrate emissions on early periods, which is not socially optimal.

${ }^{15}$ To obtain an idea on the relevance of the results, price distortions and efficiency losses based on the fringe agents' elasticities and the large agent's permit endowment range from $13 \%$ to $33 \%$ in the context of the Hot Air discussion underlying the Kyoto
} 


\section{Appendix}

\section{Solution for the Leader's Problem: Second case of Fringe Agents' Reaction Function}

Replacing $P_{t}$ by (12), the large agent's optimization program becomes:

$$
\left\{\begin{array}{l}
\min _{e_{1}} \int_{0}^{T} e^{-r t}\left\{C_{1}\left(e_{1}(t)\right)-\left\{C_{i}^{\prime}\left(e_{i}(t)\right)+\lambda(t)\right\}\left(e_{1}(t)-\bar{e}_{1}(t)\right)\right\} \mathrm{dt} \\
\dot{B}_{1}(t)=\bar{e}_{1}(t)-e_{1}(t) \\
\bar{E}=\int_{0}^{T} e_{1}(t) \mathrm{dt}+\int_{0}^{T} \sum_{i=2}^{N} e_{i}(t) \mathrm{dt} \\
B_{1}(0)=0 \\
C_{1}\left(e_{1}(0)\right)=0 \\
\forall i=2, \ldots, N
\end{array}\right.
$$

The resolution method and comments are similar to the first case:

- if $B_{1}(T)=0$, then $\mu(T)>0, \mu(t)>0$ and $B_{i}(T)=0, \forall i=2, \ldots, N$ : in case both agents clear their permits bank at the end of the period, the shadow values of a unit in the bank are positive. Both values of $\mu(t)$ and $\lambda(t)$ are known. For the large agent, the shadow value of a unit of emission in the bank measures the marginal utility of the state at time $t$ along the optimal trajectory. For fringe agents, $\lambda(t)$ reflects the highest hypothetical price at which they would be willing to pay for an additional permit at time $t$.

Rearranging as above and setting $\left\{B_{i}(T)=0, B_{1}(T)=0, \lambda(t)>0, \mu(t)>0, \forall i=2, \ldots, N\right\}$ yields:

$$
-C_{1}^{\prime}\left(e_{1}(t)\right)+\mu(t)=P(t)\left(1+\frac{1}{N-1} \varepsilon_{i}\left(e_{1}(t)-\bar{e}_{1}(t)\right)\right)-\lambda(t)
$$

This condition means that when both agents clear their permits bank in terminal period, the large agent is still able to affect negatively fringe agent's MAC. Therefore, I am able to characterize the possibility of strategic manipulation for both forms of fringe agents' reaction function.

Protocol. A journal of these numerical simulations based on data from Decaux and Ellerman (1998) and Löschel and Zhang (2002) may be obtained upon request to the author. 


\section{References}

Burniaux, J.M., 1999. How important is market power in achieving Kyoto?: An assessment based on the GREEN model. OECD Workshop on the Economic Modelling of Climate Change.

Decaux, A., Ellerman, A.D., 1998. Analysis of Post-Kyoto CO2 Emissions Trading Using Marginal Abatement Curves. MIT EPPR Report \#40.

Dockner, E.,Jorgensen, S.,Long, N. Van.,Sorger, G., 2000. Differential Games in Economics and Management Science. Cambridge Universiy Press.

Ellerman, A.D.,Wing, I.Sue., 2003. Absolute versus Intensity-Based Emission Caps. Climate Policy 3, S7S20.

Hahn, R.W., 1984. Market Power and Transferable Property Rights. Quarterly Journal of Economics 99, 753-765.

Kling, C., Rubin, J., 1997. Bankable Permits for the Control of Environmental Pollution. Journal of Public Economics 64, 101-115.

Leiby, P., Rubin, J., 2001. Intertemporal Permit Trading for the Control of Greenhouse Gas Emissions. Environmental and Resource Economics 19, 229-256.

Liski, M., Montero, J.P., 2005. A Note on Market Power in an Emissions Permit Market with Banking. Environmental and Resource Economics 31(2), Special Issue, 159-173.

Liski, M., Montero, J.P., 2006. On Pollution Permit Banking and Market Power. Journal of Regulatory Economics 29(3), 283-302.

Löschel, A., Zhang, Z.X., 2002. The Economic and Environmental Implications of the US Repudiation of the Kyoto Protocol and the Subsequent Deals in Bonn and Marrakech. FEEM Nota Di Lavoro \#23.2002.

Maeda, A., 2003. The Emergence of Market Power in Emision Rights Markets: the Role of Initial Permit Distribution. Journal of Regulatory Economics 24(3), 293-314.

Misiolek, W.S., Elder, H.W., 1989. Exclusionary Manipulation of Markets for Pollution Rights. Journal of Environmental Economics and Management 16, 156-166.

Montgomery, W.D., 1972. Markets in Licenses and Efficient Pollution Control Programs. Journal of Economic Theory 5, 395-418.

Rubin, J., 1996. A model of Intertemporal Emission Trading, Banking, and Borrowing. Journal of Environmental Economics and Management 31, 269-286.

Schennach, S.M., 2000. The Economics of Pollution Permit Banking in the Context of Title IV of the 1990 Clean Air Act Amendments. Journal of Environmental Economics and Management 40, 189-210. 